\title{
Clinical Impact of Perioperative Oral/Dental Care on Cancer Surgery Outcomes
}

\author{
TORU AOYAMA* and AYAKO TAMAGAWA* \\ Department of Surgery, Yokohama City University, Yokohama, Japan
}

\begin{abstract}
Perioperative oral/dental care has been introduced to cancer surgery patients as perioperative management using a multimodal approach. Several approaches were tested for managing perioperative oral/dental health care in this population. However, while the clinical impact of perioperative oral/dental care on patients with various types of malignancies has been evaluated, most previous studies have used and evaluated data obtained from relatively small sample sizes of fewer than 200 patients from individual institutions. Therefore, the recommendation of perioperative oral/dental care is controversial. Recent studies have shown that perioperative oral/dental care affects the cancer surgery outcomes. In addition, perioperative oral/dental care by dentistry professionals and the number of oral/dental care sessions have been shown to be important for this beneficial effect, even when patients were temporarily intubated. Further studies are, therefore, necessary to establish the optimal perioperative dental/oral care regiment and verify the beneficial effect of perioperative oral/dental care on cancer surgery outcomes. This review summarizes the background, current status, and future perspectives of perioperative oral/dental care for cancer surgery.
\end{abstract}

An estimated 14.1 million new cancer cases and 8.2 million cancer deaths occurred in 2012 worldwide (1). Surgical resection is a major method of treating cancer; however, the morbidity after cancer surgery has been reported to range from $10-50 \%$, and the complications are sometimes fatal (28). Many previous studies have shown that the development of postoperative complications increases the risk of disease

\footnotetext{
*These Authors contributed equally to this article.

Correspondence to: Toru Aoyama, Department of Surgery, Yokohama City University, 3-9 Fukuura, Kanazawa-ku, Yokohama 236-0004, Japan. Tel: +81 0457872800, e-mail: t-aoyama@lilac.plala.or.jp

Key Words: Oral care, dental care, surgical treatment, surgical complication, review.
}

recurrence in various types of malignancies (9-12). Therefore, it is important to manage the occurrence of complications before surgery and to determine the most appropriate approach of perioperative care.

Perioperative oral/dental care has been introduced in cancer surgery patients as perioperative management using a multimodal approach. Some studies have demonstrated the prophylactic effect of perioperative oral/dental care on postoperative infectious complications, such as pneumonia and surgical site infection, after cancer surgery (13-18). However, high-evidence-level studies are limited. Therefore, the recommendation of perioperative oral/dental care is controversial, and the enhanced recovery after surgery program (ERAS), as well as various cancer treatment guidelines, do not include recommendations concerning perioperative oral/dental care (19-20).

Recent studies have shown that perioperative oral/dental care affects the cancer surgery outcomes. This review summarizes the background, current status, and future perspectives of perioperative oral/dental care for cancer surgery

Perioperative oral/dental care in gastrointestinal cancer surgery. In esophageal cancer surgery, perioperative oral management exerts clinical effects on the development of postoperative pneumonia. Akutsu et al. prospectively evaluated whether dental brushing reduced the risk of postoperative pneumonia in 86 esophageal cancer patients (21). Patients were assigned to 2 groups: 41 in the control group and 45 in the dental brushing group. The patients included in the dental brushing group brushed their teeth 5 times a day (after waking up, after each meal, and before sleeping) and continued this regimen from 1 week before surgery to 1 week after surgery. The incidence of postoperative pneumonia was significantly higher in the control group than in the dental brush group (32\% versus $9 \%$, $p=0.013$ ). In addition, perioperative oral management was significantly correlated with the development of postoperative pneumonia in the multivariate analysis [odds ratio $(\mathrm{OR})=4.76$, 95\% confidence interval $(\mathrm{CI})=1.41-16.1 ; p=0.013)]$. 
Sato et al. retrospectively investigated the effectiveness of perioperative oral care for preventing postoperative pneumonia in 529 esophageal cancer surgery patients (14). Among them, 232 patients received perioperative oral care, and 297 did not. Dental examinations were performed by a dentist between the initial visit to the hospital and one week before esophagectomy. In each patient, the severity of periodontitis was classified as none, slight, or severe. Severe periodontitis was often accompanied by the need for dental extraction. Removal of plaque and dental calculi was performed in the slight group. Removal of infected tissue and tooth crown restoration were performed in patients with dental caries who did not meet the criteria for dental extraction. The dentist also explained the importance of oral care and self-care to all patients. A total 69 patients were diagnosed with grade $3 \mathrm{~B}$ postoperative pneumonia in this study. The incidence of postoperative pneumonia was significantly higher in the non-perioperative dental care group than in the perioperative dental care group $(16.5 \%$ versus $8.6 \%, p=0.008)$. The multivariate analysis showed that a lack of dental care intervention was significantly correlated with postoperative pneumonia $(\mathrm{OR}=2.517$, 95\%CI=1.376-4.775; $p=0.0025)$. The authors concluded that perioperative dental care reduced the risk of postoperative pneumonia after esophageal cancer surgery.

In a similar study by Soutome et al., the effectiveness of perioperative oral care for preventing postoperative pneumonia was investigated in 539 esophageal cancer surgery patients at 7 university hospitals (13). Of these patients, 306 received perioperative oral care, and 233 did not. The perioperative oral care group received oral care from a dentist and dental hygienist. Oral care started from the time at hospitalization and included oral health instruction, removal of dental calculi, professional mechanical tooth cleaning, removal of tongue coating with toothbrush, cleaning denture, and extraction of problem teeth. Patients were also instructed to clean their teeth using a toothbrush, interdental brush, and dental floss, followed by gargling three times per day. In addition, after surgery, the patients were asked to perform frequent gargling with water. The incidence of postoperative pneumonia was significantly higher in the non-perioperative oral care group than in the perioperative oral care group ( $25.3 \%$ versus $14.4 \%, p=0.002)$. In accordance with the results by Sato et al., Soutome et al. also showed perioperative oral care could reduce the risk of postoperative pneumonia after esophageal cancer surgery.

In colorectal cancer surgery, perioperative oral management has shown to affect the development of surgical site infection. Nobuhara et al. evaluated the clinical impact of perioperative oral management on the development of surgical site infection in 698 patients who received colorectal surgery 16. In that study, 563 patients received perioperative oral management, and 135 did not. Perioperative oral management included instruction on self-care, extraction of infected teeth, removal of dental plaques and calculi, professional mechanical teeth cleaning, removal tongue coating, and cleaning dentures. A total 68 patients experienced surgical site infection (SSI). The incidence of SSI was significantly higher in the nonperioperative oral care group than in the perioperative oral care group $(17.0 \%$ versus $8.0 \%, p=0.003)$. In addition, perioperative oral management was significantly correlated with the development of SSI in the multivariate analysis $(\mathrm{OR}=0.484,95 \% \mathrm{CI}=0.272-0.862 ; p=0.014)$. The results supported that perioperative oral care reduced the risk of SSI after colorectal cancer surgery.

Although perioperative oral/dental care did show some clinical benefits in esophageal and colorectal cancer, the clinical evidence regarding other gastrointestinal cancers, such as gastric cancer and hepatobiliary-pancreatic cancer, is still insufficient.

Perioperative oral/dental care in lung cancer surgery. In lung cancer surgery, perioperative oral management has shown a clinical effect on the development of postoperative pneumonia. Iwata et al. evaluated the clinical effect of perioperative oral management on the development of postoperative pneumonia in 721 patients who received lung resection (22). In that study, 280 patients received perioperative oral management, and 441 did not. The patients in the oral care group received oral/dental care, such as oral health maintenance, removal of dental calculi, removal of tongue coating with toothbrush, and extraction of teeth with severe periodontitis, from a dentist and dental hygienist. A total of 54 patients experienced postoperative pneumonia in that study. The incidence of postoperative pneumonia was significantly higher in the non-perioperative oral care group than in the perioperative oral care group (9.3\% versus $4.6 \%$, $p=0.002)$. In addition, perioperative oral management was significantly correlated with the development of postoperative pneumonia in the multivariate analysis $(\mathrm{OR}=2.946$, $95 \% \mathrm{CI}=1.476-5.883)$. Similar results were observed in the propensity score-matched analysis demonstrating that perioperative oral care reduced the risk of postoperative pneumonia after lung resection for lung cancer.

Perioperative oral/dental care in head and neck cancer surgery. In oral cancer surgery, there is evidence that perioperative oral management has a clinical impact on the development of wound infection. Specifically, Sato et al. investigated the effectiveness of perioperative oral health care for preventing postoperative wound infection (WI) in 66 patients with oral squamous cell carcinoma (18). In that study, 33 patients received perioperative oral health care, and 33 did not. The patients in the care group were given an oral health care plan by doctors of oral medicine and dental hygienists, including professional mechanical teeth cleaning 
(PMTC), scaling, and instructions on how to care for their oral health. Each patient in the care group received PMTC and scaling at least once before surgery. Each patient was instructed on tooth brushing using an appropriate dental brush and dental floss and on cleaning the oral mucosa and tongue using a sponge brush mouth wash. WI was observed in 14 patients in that study, and the incidence was significantly higher in the non-perioperative oral health care group than in the perioperative oral health care group (9\% versus $33 \%, p<0.025)$. The multivariate analysis showed that the lack of the oral health care intervention was significantly correlated with WI, suggesting that perioperative oral health care can reduce the risk of WI after oral cancer surgery. Treatment strategy of oral/dental care for cancer surgery.

Several approaches have been tested concerning the management of perioperative oral/dental health care in cancer surgery. While some studies have evaluated the clinical influence of perioperative oral/dental care in patients with various types of malignancies, most previous studies have used and evaluated data obtained from relatively small sample sizes of $<200$ patients from a single institution. Data obtained from small samples have many limitations, such as unspecified indications of surgery, heterogeneous populations, heterogeneous oral/dental care, and a description bias of surgical complications. Therefore, the recommendations concerning perioperative oral/dental care remain controversial. In addition, the ERAS and various types of cancer treatment guidelines do not include recommendations regarding perioperative oral/dental care $(19,20)$. To overcome such limitations associated with small sample sizes, it is necessary to evaluate oral/dental health care for cancer surgery using large data sets from individual patients collected from multiple institutes.

However, previous study findings suggested the positive results for the future study (17). It was suggested that perioperative oral/dental care by a dentist might have clinical benefits for patients undergoing cancer surgery, even in patients with temporary intubation. Previous studies that failed to show positive effects or results involved perioperative oral/dental care being performed by non-dental professionals (23-25). However, perioperative oral/dental health care is often performed by non-dentists and patients themselves; therefore, it can be difficult to remove the biofilms of the oral mucosa, teeth, and tongue as well as dental calculi without intervention by a dentistry professional. The involvement of dentists in the preoperative oral/dental care of patients undergoing cancer surgery may have some clinical effect of preventing postoperative complications. It was also suggested that the number of oral/dental care sessions is important for ensuring a good outcome (16). A previous study showed that receiving perioperative oral/dental care over two or more sessions was more effective than receiving management only once. The effect of preventing periodontal inflammation and enhancing the self-care capacity by performing oral intervention at least twice may be higher than that achieved with a single instance of intervention.

\section{Conclusion}

Although previous studies have shown the prophylactic effect of perioperative oral/dental care on postoperative complications, such as pneumonia and SSI, after cancer surgery, the recommendations regarding perioperative oral/dental care remain controversial. Further studies will be necessary in order to determine the optimal approach to perioperative dental/oral care and verify the effectiveness of perioperative oral/dental care for cancer surgery.

\section{Conflicts of Interest}

Toru Aoyama and Ayako Tamagawa have no conflicts of interest or financial ties to disclose.

\section{Authors' Contributions}

Toru Aoyama and Ayako Tamagawa both contributed to the conception and design of the study, as well as to the acquisition analysis and interpretation of data. Both authors, also contributed to drafting and revising the manuscript.

\section{Acknowledgements}

This work was supported, in part, by the non-governmental organization Yokohama Surgical Research Group, Association of Healthcare Corporation, Yoshiki Dermatology Clinic Ginza, and Social Health Corporation Foundation Chiyukai Association (Fukuoka Wajiro Hospital).

\section{References}

1 Torre LA, Bray F, Siegel RL, Ferlay J, Lortet-Tieulent J and Jemal A: Global cancer statistics, 2012. CA Cancer J Clin 65: 87-108, 2015. PMID: 25651787. DOI: 10.3322/caac.21262

2 Yoshikawa T, Rino Y, Yukawa N, Oshima T, Tsuburaya A and Masuda M: Neoadjuvant chemotherapy for gastric cancer in Japan: a standing position by comparing with adjuvant chemotherapy. Surg Today 44(1): 11-21, 2014. PMID: 23508452. DOI: $10.1007 / \mathrm{s} 00595-013-0529-1$

3 Aoyama $\mathrm{T}$ and Yoshikawa T: Adjuvant therapy for locally advanced gastric cancer. Surg Today 47(11): 1295-1302, 2017. PMID: 28251375. DOI: 10.1007/s00595-017-1493-y

4 Fujita H: History of lymphadenectomy for esophageal cancer and the future prospects for esophageal cancer surgery. Surg Today 45(2): 140-149, 2015. PMID: 24519395. DOI: 10.1007/ s00595-014-0841-4

5 Ansari D, Tingstedt B, Andersson B, Holmquist F, Sturesson C, Williamsson C, Sasor A, Borg D, Bauden M and Andersson R: Pancreatic cancer: yesterday, today and tomorrow. Future Oncol 12(16): 1929-1946, 2016. PMID: 27246628. DOI: 10.2217/fon2016-0010 
6 Uehara $\mathrm{K}$ and Nagino M: Neoadjuvant treatment for locally advanced rectal cancer: a systematic review. Surg Today 46: 161168, 2016. PMID: 26170102. DOI: 10.1007/s00595-015-1218-z

7 Watanabe A, Miyajima M, Mishina T, Tsuruta K, Takahashi Y, Maki R and Tada M: Video-assisted thoracoscopic surgery node dissection for lung cancer treatment. Surg Today 47: 1419-1428, 2017. PMID: 28285463. DOI: 10.1007/s00595-017-1494-x

8 Jemal A, Bray F, Center MM, Ferlay J, Ward E and Forman D: Global cancer statistics. CA Cancer J Clin 6: 69-90, 2011. PMID: 21296855. DOI: 10.3322/caac.20107

9 Hayashi T, Yoshikawa T, Aoyama T, Hasegawa S, Yamada T, Tsuchida K, Fujikawa H, Sato T, Ogata T, Cho H, Oshima T, Rino Y and Masuda M: Impact of infectious complications on gastric cancer recurrence. Gastric Cancer 18: 368-374, 2015. PMID: 24634097. DOI: 10.1007/s10120-014-0361-3

10 Aoyama T, Oba K, Honda M, Sadahiro S, Hamada C, Mayanagi S, Kanda M, Maeda H, Kashiwabara K, Sakamoto J, Saji S and Yoshikawa T: Impact of postoperative complications on the colorectal cancer survival and recurrence: analyses of pooled individual patients' data from three large phase III randomized trials. Cancer Med 6: 1573-1580, 2017. PMID: 28639738. DOI: $10.1002 /$ cam 4.1126

11 Aoyama T, Murakawa M, Katayama Y, Yamaoku K, Kanazawa A, Higuchi A, Shiozawa M, Morimoto M, Yoshikawa T, Yamamoto N, Rino Y, Masuda M and Morinaga S: Impact of postoperative complications on survival and recurrence in pancreatic cancer. Anticancer Res 35: 2401-2409, 2015. PMID: 25862906.

12 Kano K, Aoyama T, Yoshikawa T, Maezawa Y, Nakajima T, Hayashi T, Yamada T, Sato T, Oshima T, Rino Y, Masuda M, Cho $\mathrm{H}$ and Ogata T: The negative survival impact of infectious complications after surgery is canceled out by the response of neoadjuvant chemotherapy in patients with esophageal cancer. Ann Surg Oncol 25: 2034-2043, 2018. PMID: 29748890. DOI: 10.1245/s10434-018-6504-8

13 Soutome S, Yanamoto S, Funahara M, Hasegawa T, Komori T, Yamada SI, Kurita H, Yamauchi C, Shibuya Y, Kojima Y, Nakahara H, Oho T and Umeda M: Effect of perioperative oral care on prevention of postoperative pneumonia associated with esophageal cancer surgery: A multicenter case-control study with propensity score matching analysis. Medicine (Baltimore) 96(33): e7436, 2017. PMID: 28816937. DOI: 10.1097/MD. 0000000000007436

14 Sato Y, Motoyama S, Takano H, Nakata A, Liu J, Harimaya D, Todo N, Yoshino K, Sasaki T, Wakita A, Kawakita Y, Imai K, Saito H, Fukuda M and Minamiya Y: Esophageal cancer patients have a high incidence of severe periodontitis and preoperative dental care reduces the likelihood of severe pneumonia after esophagectomy. Dig Surg 33: 495-502, 2016. PMID: 27287475. DOI: $10.1159 / 000446927$

15 Mizuno H, Mizutani S, Ekuni D, Tabata-Taniguchi A, Maruyama T, Yokoi A, Omori C, Shimizu K, Morimatsu H, Shirakawa Y and Morita M: New oral hygiene care regimen reduces postoperative oral bacteria count and number of days with elevated fever in ICU patients with esophageal cancer. J Oral Sci 60: 536-543, 2018. PMID: 30158338. DOI: 10.2334/josnusd.17-0381

16 Nobuhara H, Yanamoto S, Funahara M, Matsugu Y, Hayashida S, Soutome S, Kawakita A, Ikeda S, Itamoto T and Umeda M: Effect of perioperative oral management on the prevention of surgical site infection after colorectal cancer surgery: A multicenter retrospective analysis of 698 patients via analysis of covariance using propensity score. Medicine (Baltimore) 97(40): e12545, 2018. PMID: 30290611. DOI: 10.1097/MD.000000 0000012545

17 Ishimaru M, Matsui H, Ono S, Hagiwara Y, Morita K and Yasunaga $\mathrm{H}$ : Preoperative oral care and effect on postoperative complications after major cancer surgery. Br J Surg 105: 16881696, 2018. PMID: 30088267. DOI: 10.1002/bjs.10915

18 Sato J, Goto J, Harahashi A, Murata T, Hata H, Yamazaki Y, Satoh A, Notani K and Kitagawa Y: Oral health care reduces the risk of postoperative surgical site infection in inpatients with oral squamous cell carcinoma. Support Care Cancer 19: 409-416, 2011. PMID: 20232086. DOI: 10.1007/s00520-010-0853-6

19 Mangram AJ, Horan TC, Pearson ML, Silver LC and Jarvis WR: Guideline for prevention of surgical site infection, 1999. Centers for Disease Control and Prevention (CDC) Hospital Infection Control Practices Advisory Committee. Am J Infect Control 27: 97-132, 1999. PMID: 10196487.

20 Lassen K, Soop M, Nygren J, Cox PB, Hendry PO, Spies C, von Meyenfeldt MF, Fearon KC, Revhaug A, Norderval S, Ljungqvist O, Lobo DN and Dejong CH: Enhanced Recovery After Surgery (ERAS) Group. Consensus review of optimal perioperative care in colorectal surgery: Enhanced Recovery After Surgery (ERAS) Group recommendations. Arch Surg 144: 961-969, 2009. PMID: 19841366. DOI: 10.1001/archsurg. 2009.170

21 Akutsu Y, Matsubara H, Shuto K, Shiratori T, Uesato M, Miyazawa Y, Hoshino I, Murakami K, Usui A, Kano M and Miyauchi H: Pre-operative dental brushing can reduce the risk of postoperative pneumonia in esophageal cancer patients. Surgery 147: 497-502, 2010. PMID: 20004439. DOI: 10.1016/ j.surg.2009.10.048

22 Iwata E, Hasegawa T, Yamada SI, Kawashita Y, Yoshimatsu M, Mizutani T, Nakahara H, Mori K, Shibuya Y, Kurita $\mathrm{H}$ and Komori T: Effects of perioperative oral care on prevention of postoperative pneumonia after lung resection: Multicenter retrospective study with propensity score matching analysis. Surgery 165: 1003-1007, 2019. PMID: 30765141. DOI: 10.1016/j.surg.2018.11.020

23 Kazaure HS, Martin M, Yoon JK and Wren SM: Long-term results of a postoperative pneumonia prevention program for the inpatient surgical ward. JAMA Surg 149: 914-918, 2015. PMID: 25054486. DOI: 10.1001/jamasurg.2014.1216

24 Cassidy MR, Rosenkranz P, McCabe K, Rosen JE and McAneny D: I COUGH: reducing postoperative pulmonary complications with a multidisciplinary patient care program. JAMA Surg 148 : 740-745, 2013. PMID: 23740240. DOI: 10.1001/jamasurg. 2013.358

25 Hiramatsu T, Sugiyama M, Kuwabara S, Tachimori Y and Nishioka M: Effectiveness of an outpatient preoperative care bundle in preventing postoperative pneumonia among esophageal cancer patients. Am J Infect Control 42: 385-388, 2014. PMID: 24679565. DOI: 10.1016/j.ajic.2013.11.017 